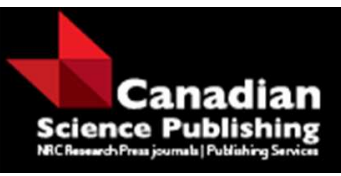

Canadian Journal of Physics

Revue canadienne de physique

\title{
Explaining Electric Conductivity Using the Particle-in-a-Box Model: Quantum Superposition is the Key
}

\begin{tabular}{|r|l|}
\hline Journal: & Canadian Journal of Physics \\
\hline Manuscript ID & cjp-2017-0158.R1 \\
\hline Danuscript Type: & Tutorial \\
\hline Complete List of Authors: & $\begin{array}{l}\text { SIVANESAN, UMASEH ; University of Toronto } \\
\text { Tsang, Kin; University of Toronto } \\
\text { Izmaylov, Artur; University of Toronto }\end{array}$ \\
\hline Keyword: & $\begin{array}{l}\text { electric conductivity, particle in a box, quantum superposition, } \\
\text { polarizability, quantum dynamics }\end{array}$ \\
\hline $\begin{array}{r}\text { Is the invited manuscript for } \\
\text { consideration in a Special }\end{array}$ & N/A \\
Issue? : & \\
\hline &
\end{tabular}

SCHOLARONE $^{\text {m }}$

Manuscripts 


\title{
Explaining Electric Conductivity Using the Particle-in-a-Box Model: Quantum Superposition is the Key
}

\author{
Umaseh Sivanesan and Kin Tsang \\ Department of Physical and Environmental Sciences, \\ University of Toronto Scarborough, Toronto, Ontario, M1C 1A4, Canada \\ Artur F. Izmaylov* \\ Department of Physical and Environmental Sciences, \\ University of Toronto Scarborough, Toronto, Ontario, \\ M1C 1A4, Canada; and Chemical Physics Theory Group, \\ Department of Chemistry, University of Toronto, \\ Toronto, Ontario, M5S 3H6, Canada
}

\begin{abstract}
Most of the textbooks explaining electric conductivity in the context of quantum mechanics provide either incomplete or semi-classical explanations that are not connected with the elementary concepts of quantum mechanics. We explain electric conductivity using the simplest model system in quantum mechanics, a particle in a box (PIB). To stimulate the particle dynamics, a linear potential tilting the bottom of the box is introduced, which is equivalent to imposing a constant electric field for a charged particle. Although the PIB model represents a closed system that cannot have a flow of electrons through the system, we consider the oscillatory dynamics of the particle probability density as the analogue of the electric current. Relating the amplitude and other parameters of the particle oscillatory dynamics with the gap between the ground and excited states of the PIB model allows us to demonstrate one of the most basic dependencies of electric conductivity on the valence-conduction band gap of the material.
\end{abstract}

\footnotetext{
*artur.izmaylov@utoronto.ca
} 


\section{INTRODUCTION}

Illustrating abstract concepts of quantum mechanics in physics education always stumbles upon inherent mathematical complexity of this theory applied to realistic systems. Thus, it is particularly beneficial for the undergraduate education to find model examples explaining complex phenomena using relatively simple mathematical consideration. In this paper we present a simple single-particle explanation of electric conductivity based on the one-dimensional particle-in-a-box (PIB) model. This model is one of the simplest in quantum mechanics and thus has enormous potential for explaining fundamental principles and phenomena in a way that is accessible to an undergraduate student.

Electric conductivity is an inherently quantum phenomenon because it depends on the energy level structure present in materials. Although electron orbital energies in periodic materials form continuous bands, the energy gap between occupied orbitals (valence band) and unoccupied orbitals (conduction band) can be finite. This gap defines the conductive properties of any material: the band gap of conductive materials is small for semi-conductors or even zero for metals, while nonconductive materials or insulators have a large gap.

Electric conductivity is a dynamical phenomenon and in quantum mechanics it must be represented by the dynamics of a non-stationary or superposition state. Only in a superposition state the system probability density can be time-dependent, and thus, the physically observable probability of finding the system in a particular region of space changes with time. Hence, if one would like to provide a simple, idealized, zero-temperature explanation within quantum mechanics without going into quantum statistical thermodynamics, the system needs to be considered in a superposition state. Moreover, since the superposition is the key to electronic motion under the influence of electric field, its creation should be more pronounced when the gap between ground and excited levels is smaller. On the other hand increasing the gap should reduce the potency of the electric field to create a superposition state.

Unfortunately, in the desire for simplicity, most of the textbooks go too far passing by the concept of the superposition state and produce awkward explanations, where under the electric field potential, an electron can move and at the same time be in a stationary state. [1, 2] This is of course a misleading oversimplification, because the probability density for any object in a stationary state is time-independent. 
Some solid-state textbooks $[3,4]$ provide an explanation that involves electronic superposition states or wave packets built as a linear combination of one-electron Bloch functions obtained for periodic potentials. Of course, such explanations would not be possible to incorporate in undergraduate quantum mechanics courses without significant detours into the consideration of periodic systems.

The key element of our PIB consideration is the superposition state emerging under the influence of an electric field, modelled as a sudden tilt of the potential box's bottom. This superposition state gives rise to the quantum dynamics necessary for particle transport within the box. Properties of this superposition state are related to the gap between occupied and unoccupied energy levels, thus providing a simple explanation of the gap-conduction relation in real materials. There are a few simplifications separating the dynamics of our model system from the conductance dynamics in the real material: 1) only one electron is considered and thus electron-electron and electron-nuclear interactions are neglected; 2) the PIB model represents a closed system, and its dynamics cannot produce steady particle current; instead, the particle's movement has an oscillatory character. These simplifications are not essential for illustrating the gap-conductance relation if we associate the conductance with various dynamical properties of a single-particle probability density.

This view of conduction can be taught at the elementary level of quantum mechanics for undergraduate students. All illustrations provided in this paper can be demonstrated in the class or given as separate projects for advanced undergraduate or even graduate courses.

The rest of the paper is organized as follows. In Sec. II.A we consider the PIB model and the introduction of a constant electric field. Section II.B-D describe various properties characterizing dynamics in the model using perturbative considerations. We illustrate all discussions in Sec. III by simulating the dynamical quantities employing the variational method for the PIB model with an inclined bottom. For simplicity of involved expressions, atomic units are used throughout this paper. 


\section{THEORY}

\section{A. Particle in a box model}

To establish notation let us introduce a particle of unit charge with mass $m$ in a box of size $L$ with infinite potential walls, then the Hamiltonian is

$$
\begin{aligned}
& H_{0}(x)=-\frac{1}{2 m} \frac{d^{2}}{d x^{2}}+V_{0}(x), \\
& V_{0}(x)=\left\{\begin{array}{l}
0, x \in[0, L] \\
\infty, x \notin[0, L] .
\end{array}\right.
\end{aligned}
$$

$H_{0}$ defines the time-independent Schrödinger equation for the stationary states

$$
H_{0}(x) \psi_{n}^{(0)}(x)=E_{n}^{(0)} \psi_{n}^{(0)}(x)
$$

where the eigenenergies and eigenfunctions are

$$
\begin{aligned}
E_{n}^{(0)} & =\frac{n^{2} \pi^{2}}{2 m L^{2}}, \\
\psi_{n}^{(0)}(x) & =\sqrt{\frac{2}{L}} \sin \left(\frac{n \pi x}{L}\right),
\end{aligned}
$$

and they are enumerated by the subscript $n$, where $n=1$ corresponds to the ground state.

We will use the ground state as the initial state of our system in the absence of the electric field. To model the electric field that creates a bias, we add to PIB's Hamiltonian a linear potential within the box

$$
V(x)=\left\{\begin{array}{l}
-\mathcal{E} x, x \in[0, L] \\
\infty, x \notin[0, L]
\end{array}\right.
$$

so that the total system Hamiltonian with the electric field becomes

$$
H(x)=H_{0}(x)+V(x)
$$

This linear potential can be seen as a result of applying a constant electric field $\mathcal{E}$ because the electric potential $V(x)$ entering the Hamiltonian for a charged particle will be exactly $-\mathcal{E} x$.

Upon a sudden turn-on of the electric field, our original state $\psi(x, t=0)=\psi_{1}^{(0)}(x)$ is not a stationary state of $H(x)$, it is instead a superposition of $H(x)$ 's eigenstates and thus 
undergoes dynamics. The simplest way to obtain this dynamics is using the eigenstates of $H(x)$ defined by

$$
H(x) \psi_{n}(x)=E_{n} \psi_{n}(x)
$$

to solve the time-dependent Schrödinger equation (TDSE). Expanding the initial state

$$
\begin{aligned}
\psi(x, 0) & =\psi_{1}^{(0)}(x) \\
& =\sum_{n=1}^{\infty} c_{n} \psi_{n}(x)
\end{aligned}
$$

as a linear combination of $\psi_{n}(x)$ with coefficients

$$
c_{n}=\int_{0}^{L} d x \psi_{n}^{*}(x) \psi_{1}^{(0)}(x)
$$

allows us to write a solution of TDSE as

$$
\psi(x, t)=\sum_{n=1}^{\infty} c_{n} \psi_{n}(x) e^{-i E_{n} t} .
$$

This wave function has a time-dependent probability distribution

$$
|\psi(x, t)|^{2}=\sum_{n, k} c_{n}^{*} c_{k} \psi_{n}^{*}(x) \psi_{k}(x) e^{i\left(E_{n}-E_{k}\right) t} .
$$

The key element of this time-dependence is the superposition character of the original wave function, thereby leading to time-dependent exponents in Eq. (13). In contrast, if $\psi(x, 0)$ consisted of a single eigenstate $\psi_{n}(x)$, its time-dependence $\psi(x, t)=\psi_{n}(x) \exp \left(-i E_{n} t\right)$ would not be present in the probability density $|\psi(x, t)|^{2}=\left|\psi_{n}(x)\right|^{2}$.

In our model, no matter how small the electric field is, $\psi(x, 0)=\psi_{1}^{(0)}(x)$ will always be a non-stationary function for the total Hamiltonian. Thus, the dynamics will always be present; the only question is whether the changes in localization of the probability distribution over time are significant. It is clear from Eq. (13) that for significant dynamics, the amplitudes of the cross terms $c_{n}^{*} c_{k}(n \neq k)$ must be large.

To obtain the $c_{n}$ 's and $E_{n}$ 's needed for simulating Eq. (13) one can use the variational approach, which searches for extrema of the energy functional

$$
E=\frac{\langle\psi|H| \psi\rangle}{\langle\psi \mid \psi\rangle}
$$


with respect to variation of a trial wave function $\psi(x)$. The simplest form of the trial wave function is a linear parameterization

$$
\psi(x)=\sum_{n=1}^{N} C_{n} \psi_{n}^{(0)}(x),
$$

where $C_{n}$ 's can be varied to find extrema of $E$. This variation leads to a system of $N$ equations obtained from the $\frac{\partial E}{\partial C_{n}}=0$ conditions which is equivalent to the eigenvalue problem

$$
\mathbf{H C}=E \mathbf{C}
$$

where $\mathbf{H}$ is the Hamiltonian matrix with the elements

$$
\begin{aligned}
H_{n k} & =\int_{0}^{L} \psi_{n}^{(0) *}(x) \hat{H} \psi_{k}^{(0)}(x) d x \\
& = \begin{cases}\frac{4 \mathcal{E} n k L\left[1-(-1)^{n+k}\right]}{\pi^{2}(n-k)^{2}(n+k)^{2}}, & n \neq k \\
\frac{n^{2} \pi^{2}}{2 m L^{2}}-\frac{\mathcal{E} L}{2}, & n=k,\end{cases}
\end{aligned}
$$

and $\mathbf{C}=\left(C_{1}, C_{2}, \ldots C_{N}\right)^{T}$ is an eigenvector corresponding to an eigen-energy $E$. There are $N$ eigenvectors and $N$ eigenenergies in total; the $n$ 'th $E$ corresponds to an approximation to

$E_{n}$ in Eq. (8), and $C_{1}$ for the $n$ 'th $\mathbf{C}$ is equal to $\int_{0}^{L} d x \psi_{1}^{(0) *}(x) \psi_{n}(x)$. Since all quantities in our problem are real these coefficients are also equal to $c_{n}$ in Eqs. (10) and (11). Therefore, solving the eigenvalue problem in Eq. (16) gives all needed quantities to construct the dynamics of the initial distribution under the influence of the sudden turn-on of the constant electric field using Eq. (13).

\section{B. Dynamics of probability density}

To obtain some qualitative insights in what determines the probability density dynamics, we will consider which system parameters affect $c_{n}$ coefficients. According to Eq. (13) only the terms that are products of coefficients from different states contribute to the timedependence, therefore to have more pronounced dynamics more coefficients should have non-negligible values. We will use time-independent perturbation theory (TIPT) as our main tool in this analysis. TIPT formulates eigenstates of $H(x)$ as a series

$$
\psi_{n}(x)=\psi_{n}^{(0)}(x)+\psi_{n}^{(1)}(x)+\psi_{n}^{(2)}(x)+\ldots
$$


where $\psi_{n}^{(0)}(x)$ are eigenfunctions of the $H_{0}$ Hamiltonian and $\psi_{n}^{(k)}(x)$ are higher order perturbative corrections to $\psi_{n}^{(0)}(x)$. If we substitute this expansion in Eq. (11) we can obtain the corresponding expansion for $c_{n}$

$$
\begin{aligned}
c_{n} & =c_{n}^{(0)}+c_{n}^{(1)}+c_{n}^{(2)}+\ldots \\
c_{n}^{(k)} & =\int_{0}^{L} d x\left[\psi_{n}^{(k)}(x)\right]^{*} \psi_{1}^{(0)}(x) .
\end{aligned}
$$

For weak electric fields we can neglect all terms beyond the first order. Note that due to the orthogonality of the zeroth-order functions, we have only the zeroth-order term for $n=1$. For $n \neq 1$, the first nontrivial term is $c_{n}^{(1)}$. In the non-degenerate case corresponding to $H_{0}$, TIPT provides the following expression for $\psi_{n}^{(1)}(x)$,

$$
\psi_{n}^{(1)}(x)=\sum_{k \neq n} \frac{\left\langle\psi_{k}^{(0)}|V| \psi_{n}^{(0)}\right\rangle}{E_{n}^{(0)}-E_{k}^{(0)}} \psi_{k}^{(0)}(x),
$$

Substituting this equation into Eq. (21) and using the orthogonality condition $\left\langle\psi_{k}^{(0)} \mid \psi_{1}^{(0)}\right\rangle=$ $\delta_{k 1}$ gives

$$
c_{n}^{(1)}=\frac{\left\langle\psi_{n}^{(0)}|V| \psi_{1}^{(0)}\right\rangle}{E_{1}^{(0)}-E_{n}^{(0)}}, n \neq 1 .
$$

This expression clearly shows that $c_{n}^{(1)}$ are inversely proportional to the gap between ground and $n$ 'th state $E_{1}^{(0)}-E_{n}^{(0)}$. Both numerator and denominator of this expression can be evaluated analytically for our model

$$
\begin{aligned}
\left\langle\psi_{n}^{(0)}|V| \psi_{1}^{(0)}\right\rangle & =\frac{4 n \mathcal{E} L\left[(-1)^{n}+1\right]}{\pi^{2}\left(n^{2}-1\right)^{2}}, \\
E_{1}^{(0)}-E_{n}^{(0)} & =\frac{\pi^{2}\left(1-n^{2}\right)}{2 m L^{2}} .
\end{aligned}
$$

Assembling all the components we obtain

$$
c_{n}^{(1)}=\frac{-8 m n \mathcal{E} L^{3}\left[(-1)^{n}+1\right]}{\pi^{4}\left(n^{2}-1\right)^{3}}, n \neq 1 .
$$

To characterize a non-stationary character of the initial distribution, one can use the sum over weights of all excited states

$$
\begin{aligned}
\omega_{\mathrm{exc}} & =\sum_{n=2}^{\infty}\left|c_{n}\right|^{2} \approx \sum_{n=2}^{\infty}\left|c_{n}^{(1)}\right|^{2} \\
& =\sum_{n=2}^{\infty} \frac{64 m^{2} \mathcal{E}^{2} n^{2} L^{6}\left[(-1)^{n}+1\right]^{2}}{\pi^{8}\left(n^{2}-1\right)^{6}} .
\end{aligned}
$$


Here, all odd $n$ terms are zero, thus only the even terms $(n=2 j)$ need to be considered

$$
\begin{aligned}
\omega_{\mathrm{exc}} & =\frac{1024 m^{2} \mathcal{E}^{2} L^{6}}{\pi^{8}} \sum_{j=1}^{\infty} \frac{j^{2}}{\left(4 j^{2}-1\right)^{6}} \\
& =\frac{\left(2 \pi^{4}+5 \pi^{2}-210\right) m^{2} \mathcal{E}^{2} L^{6}}{240 \pi^{6}} .
\end{aligned}
$$

The infinite sum has been determined by the Mathematica program. [5] Therefore, perturbation theory predicts growth of $\omega_{\text {exc }}$ as $m^{2} \mathcal{E}^{2} L^{6}$.

\section{Polarizability}

An alternative way to characterize the mobility of the initial distribution is to calculate the polarizability of the system. The polarizability of the ground state is the second derivative of the energy with respect to the electric field

$$
\alpha=\left.\frac{d^{2} E_{1}}{d \mathcal{E}^{2}}\right|_{\mathcal{E}=0} .
$$

TIPT is the exactly right tool to obtain such derivatives.[6] According to TIPT, the total energy of the ground state is

$$
E_{1}=E_{1}^{(0)}+E_{1}^{(1)}+E_{1}^{(2)}+\ldots
$$

where the $k^{\prime}$ th order $E^{(k)}$ is proportional to $\mathcal{E}^{k}$, therefore, the polarizability is the second order correction to the energy in TIPT

$$
\alpha=\frac{d^{2} E_{1}^{(2)}}{d \mathcal{E}^{2}}=\frac{d^{2}}{d \mathcal{E}^{2}} \sum_{n \neq 1} \frac{\left|\left\langle\psi_{1}^{(0)}|V| \psi_{n}^{(0)}\right\rangle\right|^{2}}{E_{1}^{(0)}-E_{n}^{(0)}} .
$$

Thus the system is more polarizable when the gaps between the ground and excited states are low. Using Eqs. (24) and (25) the expression for polarizability can be further simplified as

$$
\alpha=\frac{-64 m L^{4}}{\pi^{6}} \sum_{n \neq 1} \frac{n^{2}\left[(-1)^{n}+1\right]^{2}}{\left(n^{2}-1\right)^{5}}
$$

This infinite sum can be evaluated by the Mathematica program[5]

$$
\alpha=\frac{\left(\pi^{2}-15\right)}{12 \pi^{4}} m L^{4} .
$$

Therefore, the system polarizability grows linearly with the particle mass and quartically with the size of the box. 


\section{Charge mobility and oscillation amplitude}

A more advanced but closely-related characterization of the system dynamics can be done using the conventional definition of the electron mobility, $\mu_{e}=v_{e} / \mathcal{E}$, where

$$
v_{e}=\frac{d}{d t}\langle\psi(t)|x| \psi(t)\rangle
$$

is the average electron velocity under the influence of the electric field $\mathcal{E}$. Employing the Ehrenfest theorem[7] it is easy to show that for our system

$$
\begin{aligned}
\frac{d}{d t}\langle\psi(t)|x| \psi(t)\rangle & =\frac{\langle\psi(t)|p| \psi(t)\rangle}{m} \\
\frac{d}{d t}\langle\psi(t)|p| \psi(t)\rangle & =-\left\langle\psi(t)\left|\frac{d V}{d x}\right| \psi(t)\right\rangle=\mathcal{E} .
\end{aligned}
$$

The electron mobility is related to the electron conductivity $(\sigma)$ with the simple relation $\sigma=n e \mu_{e}$, where $n$ is the number of electrons and $e$ their charge. The equations for the mobility and conductivity are appropriate for open systems where the steady flow of electrons is possible. In our closed system, the dynamics will always have an oscillatory nature. To characterize its extent we will take the maximum value over the period

$$
\begin{aligned}
\mu_{e} & =\max _{t} \frac{d}{d t}\langle\psi(t)|x| \psi(t)\rangle / \mathcal{E} \\
& =\frac{1}{m \mathcal{E}} \max _{t}\langle\psi(t)|p| \psi(t)\rangle
\end{aligned}
$$

In addition, since we deal with the oscillatory motion, the mobility can be characterized not only by velocity but also with the amplitude of the oscillation

$$
A=\max _{t}|\langle\psi(0)|x| \psi(0)\rangle-\langle\psi(t)|x| \psi(t)\rangle| / L
$$

To understand what parameters determine $\mu_{e}$ and $A$ we will employ time-dependent perturbation theory (TDPT) for the wave function time dependence

$$
\psi(x, t)=\psi^{(0)}(x, t)+\psi^{(1)}(x, t)+\ldots
$$


Introducing the TDPT expansion for the wave function in Eqs. (40) and (41) and restricting consideration up to the 1st order we obtain the following equations

$$
\begin{aligned}
\mu_{e}^{(0+1)} & =\frac{1}{m \mathcal{E}} \max _{t}\left[\left\langle\psi^{(0)}(t)|p| \psi^{(1)}(t)\right\rangle+\text { h.c. }\right] \\
& =\frac{2}{m \mathcal{E}} \max _{t} \operatorname{Re}\left[\left\langle\psi^{(0)}(t)|p| \psi^{(1)}(t)\right\rangle\right] \\
A^{(0+1)} & =\frac{1}{L} \max _{t} \mid\left\langle\psi^{(0)}(t)|x| \psi^{(1)}(t)\right\rangle+\text { h.c. } \mid . \\
& =\frac{2}{L} \max _{t} \operatorname{Re}\left[\left\langle\psi^{(0)}(t)|x| \psi^{(1)}(t)\right\rangle\right],
\end{aligned}
$$

where h.c. is the Hermitian conjugate, and the following easily verifiable relations are used

$$
\begin{aligned}
\left\langle\psi^{(0)}(t)|p| \psi^{(0)}(t)\right\rangle & =0, \\
\langle\psi(0)|x| \psi(0)\rangle & =\left\langle\psi^{(0)}(t)|x| \psi^{(0)}(t)\right\rangle .
\end{aligned}
$$

Following the 1 st order TDPT, the $\psi^{(1)}(x, t)$ will be given by

$$
\begin{aligned}
\psi^{(1)}(x, t) & =\sum_{n \neq 1} d_{n}^{(1)}(t) \psi_{n}^{(0)}(x) e^{-i E_{n}^{(0)} t} \\
d_{n}^{(1)}(t) & =i \mathcal{E} \int_{0}^{t}\left\langle\psi_{1}^{(0)}|x| \psi_{n}^{(0)}\right\rangle e^{i\left(E_{n}^{(0)}-E_{1}^{(0)}\right) \tau} d \tau \\
& =\frac{\mathcal{E}\left\langle\psi_{1}^{(0)}|x| \psi_{n}^{(0)}\right\rangle}{E_{n}^{(0)}-E_{1}^{(0)}}\left(e^{i\left(E_{n}^{(0)}-E_{1}^{(0)}\right) t}-1\right)
\end{aligned}
$$

Combining all the terms gives

$$
\begin{aligned}
\psi^{(1)}(x, t)= & \sum_{n \neq 1} \psi_{n}^{(0)}(x) \frac{\mathcal{E}\left\langle\psi_{1}^{(0)}|x| \psi_{n}^{(0)}\right\rangle}{E_{n}^{(0)}-E_{1}^{(0)}} \\
& \times\left(e^{-i E_{1}^{(0)} t}-e^{-i E_{n}^{(0)} t}\right)
\end{aligned}
$$

Using this in combination with $\psi^{(0)}(x, t)=e^{-i E_{1}^{(0)} t} \psi_{1}^{(0)}(x)$ allows us to express the mobility as a series

$$
\begin{aligned}
\mu_{e}^{(0+1)}= & \frac{32 L^{2}}{\pi^{4}} \max _{t}\left\{\sum_{n \neq 1}^{\infty} \frac{n^{2}\left[(-1)^{n}+1\right]^{2}}{\left(n^{2}-1\right)^{4}}\right. \\
& \left.\times \sin \left[\frac{t \pi^{2}\left(n^{2}-1\right)}{2 m L^{2}}\right]\right\}
\end{aligned}
$$

As the degree of $n$ is much higher in the denominator than the numerator, and all odd $n$ terms are zero, the first non-zero term, $n=2$, will have the largest effect. If we take only this term, we can maximize the sine function by choosing

$$
t=\frac{m L^{2}(4 k+1)}{3 \pi}, k \in \mathbb{Z} .
$$


Using this $t$ in the $n=2$ term we obtain an estimate for the maximum charge mobility,

$$
\mu_{e}^{(0+1)} \approx \frac{512 L^{2}}{81 \pi^{4}}
$$

This expression is independent of mass, showing that while the charge mobility varies quadratically with the size of the box, it is not affected by the mass of the particle. This mass independence is somewhat counterintuitive from the classical point of view because one would expect heavier particles to be slower and lighter particles to be faster. However, in the PIB model, gaps between the ground and excited states are inversely proportional to the mass [Eq. (25)], which generally makes the mobility higher as mass increases. On the other hand, the mass dependence that is in Eq. (40) provides the opposite trend. The two tendencies compensate each other, removing the mass dependence from the mobility.

Similar to the mobility, the oscillation amplitude can be expressed as a series

$$
\begin{aligned}
A^{(0+1)}= & \frac{64 \mathcal{E} m L^{3}}{\pi^{6}} \max _{t}\left[\sum_{n \neq 1}^{\infty} \frac{n^{2}\left[(-1)^{n}+1\right]^{2}}{\left(n^{2}-1\right)^{5}}\right. \\
& \left.\times\left(1-\cos \left(\frac{t \pi^{2}\left(n^{2}-1\right)}{2 m L^{2}}\right)\right)\right] .
\end{aligned}
$$

The terms in the sum decrease in magnitude as $n$ increases, so the $n=2$ term will have the greatest contribution to the overall sum. For this term, the value of $t$ that maximizes the $n=2$ term is

$$
t=\frac{2 m L^{2}(2 k+1)}{3 \pi}, k \in \mathbb{Z}
$$

This $t$ gives an approximate value for the maximum amplitude

$$
A^{(0+1)} \approx \frac{2048 \mathcal{E} m L^{3}}{243 \pi^{6}} .
$$

In contrast with the mobility, the amplitude not only grows with $L$ but also increases linearly with $m$ and $\mathcal{E}$.

\section{NUMERICAL ILLUSTRATION}

All quantities that we have considered in connection with the electron transport in the PIB system are inversely proportional to the gap between the ground and excited states. This gap is determined by two parameters: $m$ and $L$ [Eq. (25)]. Here we illustrate the dependence of 

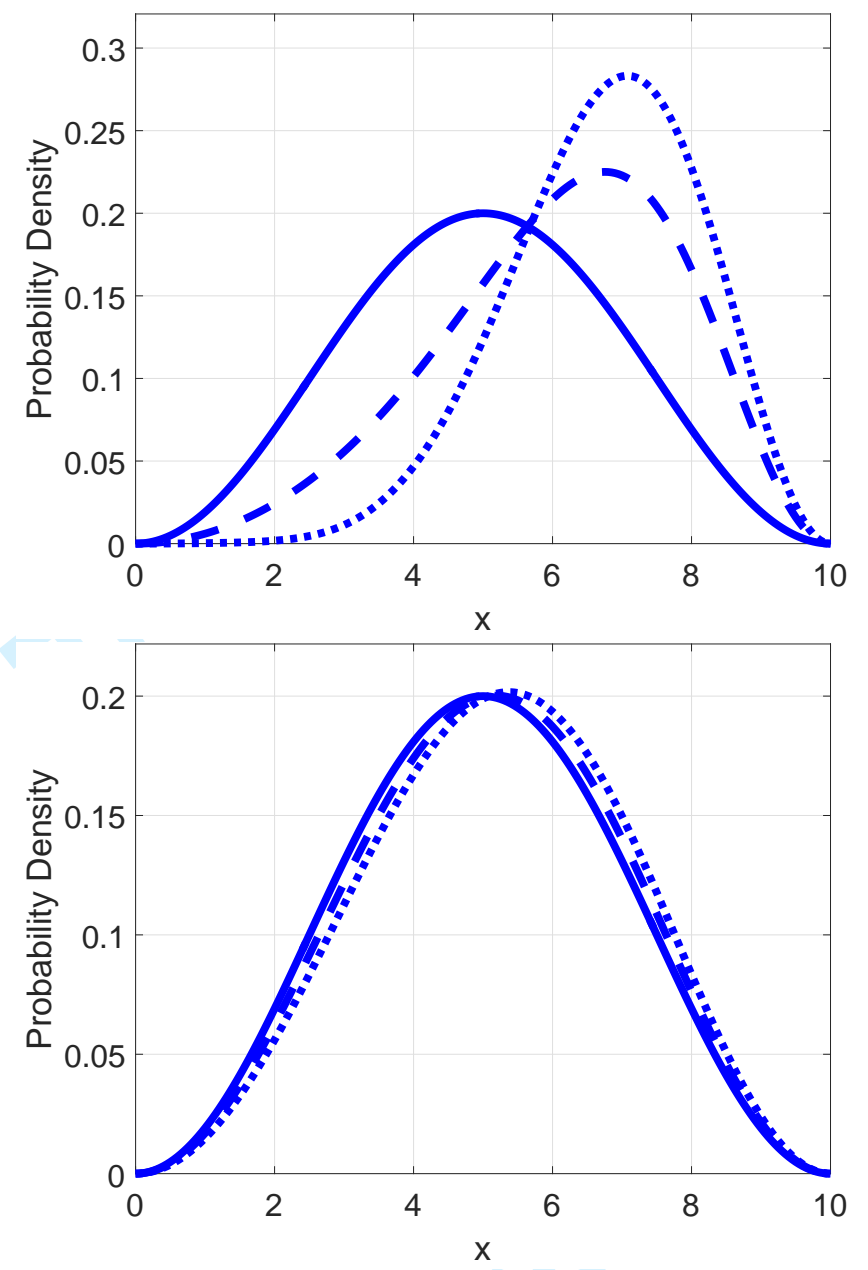

FIG. 1. Probability density as a function of the $x$-coordinate for $m=0.5$ (upper plot), $m=0.05$ (lower plot) at different times: $t=0$ (solid), $t=t_{\max } / 2$ (dashed), $t=t_{\max }$ (dotted). $L=10$, $\mathcal{E}=0.05, t_{\max } \approx 200 \mathrm{~m} /(3 \pi)$, and 20 eigenfunctions were used for both systems.

the considered properties on these two parameters using the variational approach to obtain eigenstates of the PIB problem with a finite field. Results of variational calculations will be compared to the perturbative estimates obtained in the Theory section.

a. Probability density oscillations: One of the simplest demonstrations of the relation between conductivity and the gap is to observe the probability density dynamics for two particles of different masses (see Fig. 1). We build these dynamics using Eq. (13) to evaluate $|\psi(x, t)|^{2}$ and plot it as a function of $x$ for a few times. The lower mass system has a larger gap (see Eq. (25)) and oscillations of its probability density are diminished compare to those in the heavy mass system, where the gap is much smaller.

According to Eq. (58), the amplitude of oscillations is dependent on $\mathcal{E}, m$, and $L$, and https://mc06.manusqrijptcentral.com/cjp-pubs 
Fig. 2 summarizes our comparison of the amplitude obtained using variational and perturbative expressions. As evident from Fig. 2, the variational method's results agree with Eq. (58) from the perturbative approach, assuming the perturbation is not large. Both approaches reveal that amplitude increases with $L$, since increasing $L$ decreases the gap and makes system more susceptible to oscillations. Note that this does not simply happen because the larger box gives the particle more room to move; such logic would be incorrect because we consider the amplitude normalized by the size of the box [Eq. (41)]. One can also see an obvious trend of increased amplitudes for heavier masses, which again stems from the reduction of the gap for increased mass, described by Eq. (25). Finally, there is a dependence on $\mathcal{E}$, as a larger applied potential causes a greater oscillation amplitude.

b. Eigenstates for the PIB model with an inclined bottom: To understand quantum interference of what states leads to the oscillations depicted in Fig. 1 we consider the eigenstates of $H$ in Eq. (7). Note that the key role in spatial extent of the oscillations is the spatial distribution of probability density for individual states, because if all states forming a superposition are localized in a certain region of space, the interference dynamics cannot leave that region. Figure 3 compares the three lowest eigenstates for the PIB model with and without inclination of the bottom. It is clear that the inclination shifts the maximum of the probability distribution for the ground state closer to the region of lower potential. Interestingly, maxima of the probability density for the excited states shift the other way. This can be understood considering that all eigenstates must be mutually orthogonal. Therefore, if excited states followed the ground state they would need to have an increasing number of nodes in the region of low potential to be orthogonal to the ground state, which would increase their kinetic energy. Instead, they shift the other way in order to avoid introducing excessive curvature.

c. Weight of excited states: Another important component of the oscillatory dynamics is how many excited states are substantially involved in the superposition. Examining these states for the PIB model with an inclined bottom shown that excited states are generally shifted in the opposite side of the box compare to the shift of the ground state. However, if we do not have substantial weights of excited states in the superposition, their localization will not be able to play a role in dynamics. To characterize how the total weight of excited states changes within the initial state $\psi(x, 0)=\psi_{1}^{(0)}(x)$ after the field is turned on, we evaluate $\omega_{\text {exc }}=\sum_{n=2}^{20}\left|c_{n}\right|^{2}$ dependence on $L$ and $m$ using the variational approach [see Fig. 4]. Going 

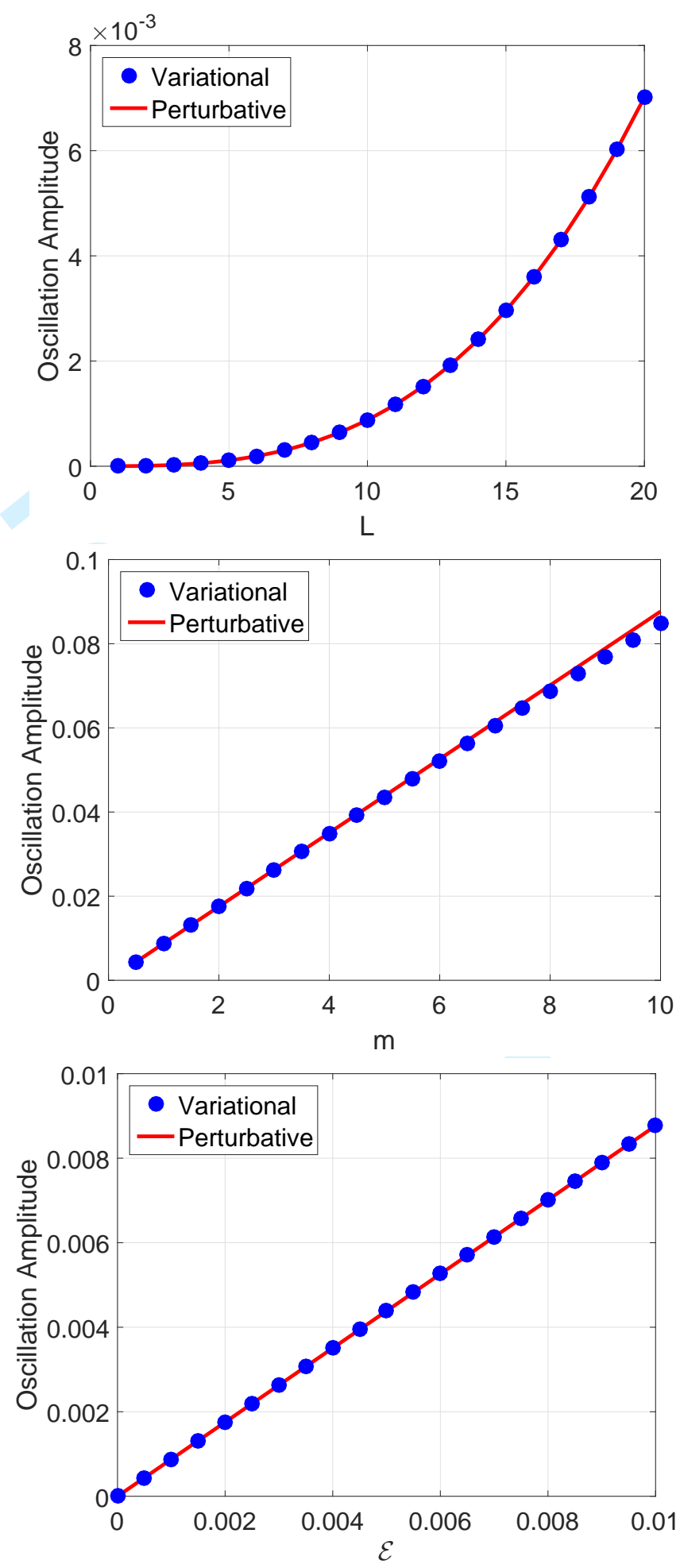

FIG. 2. Maximum amplitude as a function of $L$ (upper plot), $m$ (middle plot), and $\mathcal{E}$ (lower plot). Fixed values were $m=0.1, \mathcal{E}=0.001$, and $L=10$. 

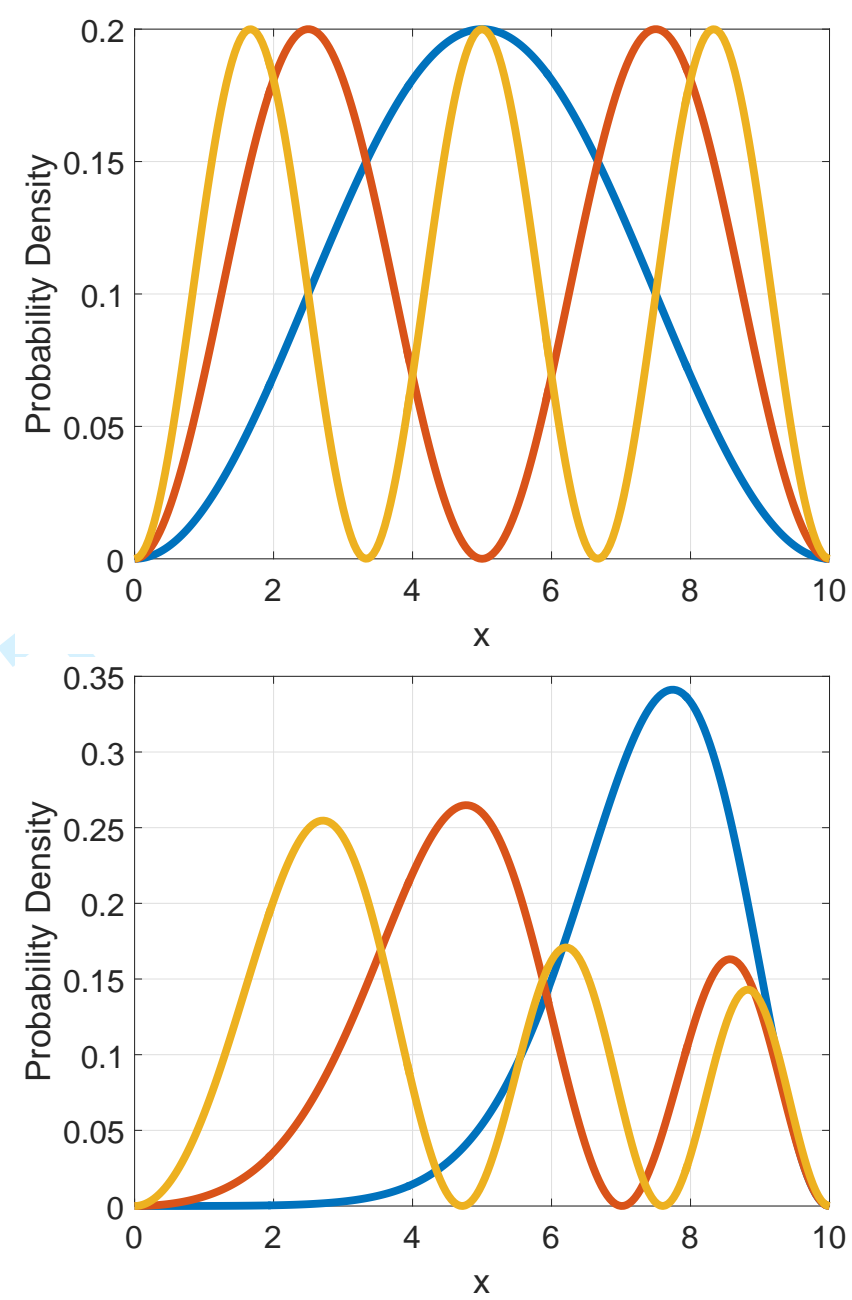

FIG. 3. The probability densities for the first three eigenfunctions of PIB problems, where $m=1$ and $L=10$ : ground (blue), first (red) and second (green) excited states. The top (bottom) plot corresponds to eigenstates of $H_{0}(H$ with $\mathcal{E}=0.1)$.

beyond 20 states in the $\omega_{\text {exc }}$ expansion does not produce an appreciable contribution in the range of reported parameters. The perturbative estimate of $\omega_{\text {exc }}$ in Eq. (30) is in very good agreement with variational results. As the electric field strength increases, the first order perturbative estimate in Eq. (30) becomes less accurate [Fig. 4] but maintains qualitatively correct trends. The origin of the growth of $\omega_{\text {exc }}$ with $L$ and $m$ is a gap reduction between the ground and excited states.

d. Polarizability: This quantity characterizes the response of the system to an infinitesimal electric field. Perturbation theory provides exact polarizabilities and to illustrate this numerically we compared perturbative expressions [Eq. (35)] with estimates obtained via 

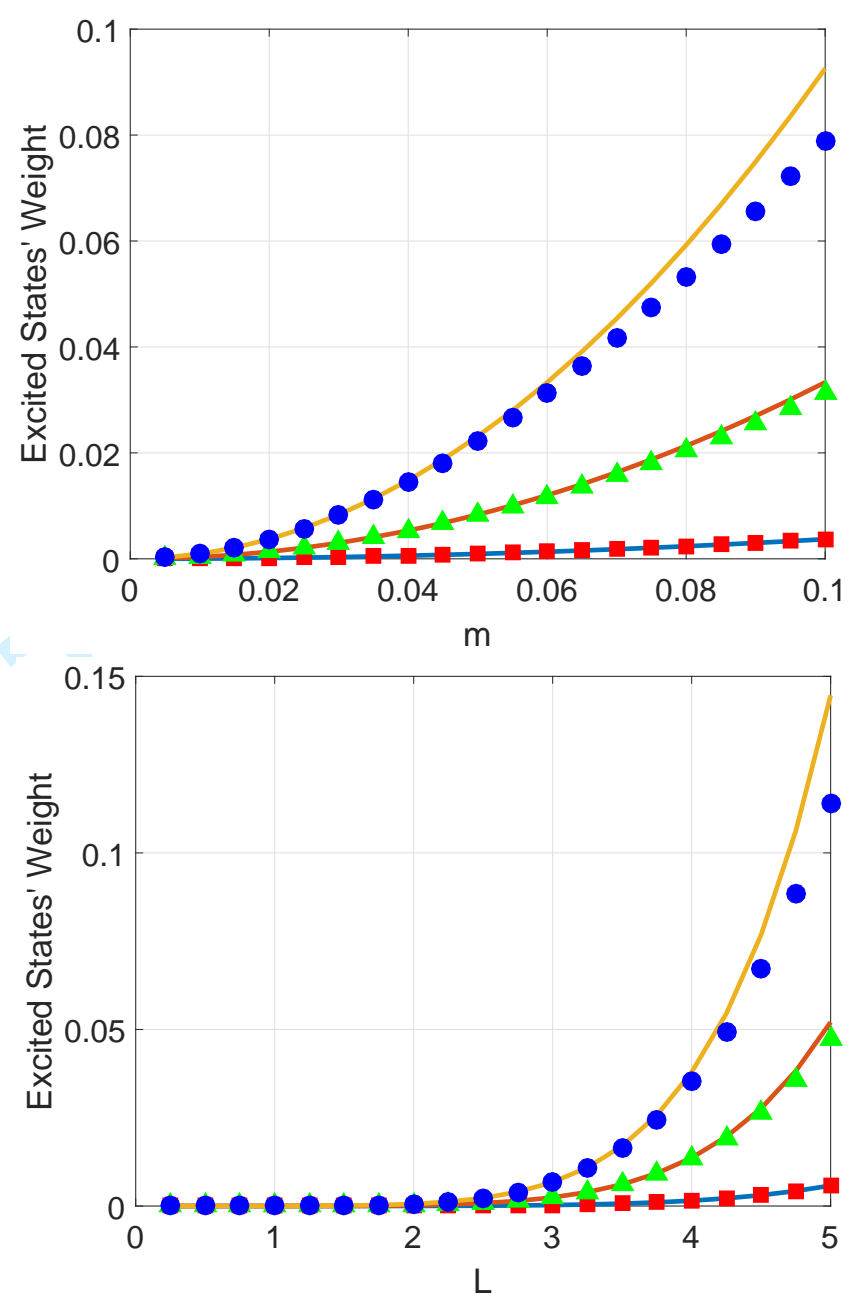

FIG. 4. The weight of excited states in the initial state $\left(\omega_{\text {exc }}\right)$ as a function of the particle mass (top panel, $L=10$ ) and as a function of the box size (bottom panel, $m=1$ ). Dots (solid lines) correspond to variational (perturbative [Eq. (30)]) results for different field strengths: $\mathcal{E}=0.05$ squares, $\mathcal{E}=0.1$ triangles, and $\mathcal{E}=0.15$ circles.

numerical second derivatives of variational energies. The energy differentiation was accomplished via the central finite differencing scheme within the eighth-order expression[8]

$$
\begin{aligned}
\alpha \approx & \left(\frac{-1}{560} E(-4 \mathcal{E})+\frac{8}{315} E(-3 \mathcal{E})-\frac{1}{5} E(-2 \mathcal{E})+\frac{8}{5} E(-\mathcal{E})\right. \\
& -\frac{205}{72} E(0)+\frac{8}{5} E(\mathcal{E})-\frac{1}{5} E(2 \mathcal{E})+\frac{8}{315} E(3 \mathcal{E}) \\
& \left.-\frac{1}{560} E(4 \mathcal{E})\right) / \mathcal{E}^{2}+O\left(\mathcal{E}^{8}\right),
\end{aligned}
$$

where $E(\mathcal{E})$ is the ground state variational energy obtained at the $\mathcal{E}$ field value, $\mathcal{E}=10^{-4}$ a.u. was used. Figure 5 shows that perturbative and variational polarizability results are 

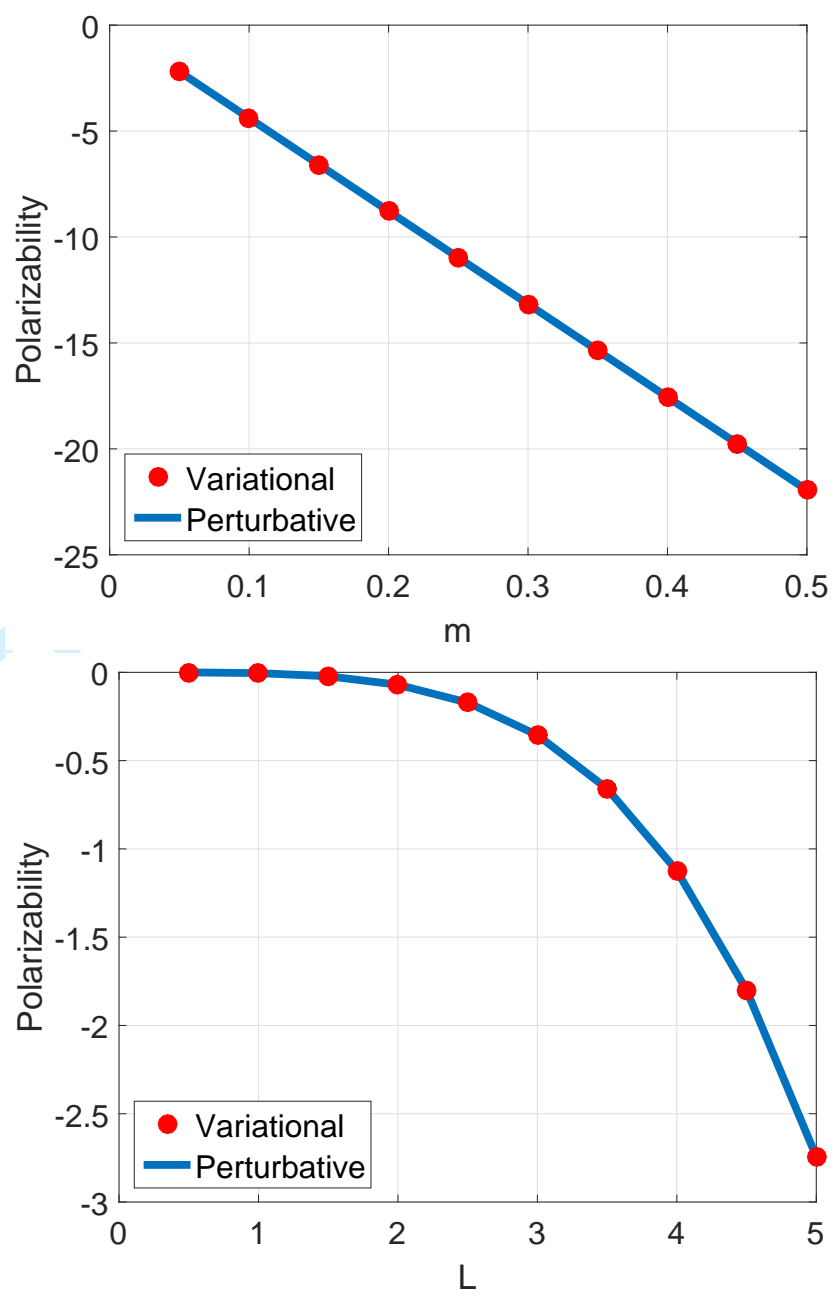

FIG. 5. Polarizability as a function of the particle mass (top panel, $L=10$ ) and as a function of the box size (bottom panel, $m=1$ ). Dots (solid lines) correspond to the variational (perturbative [Eq. (35)]) approach.

seemingly indistinguishable, both approaches display linear dependence on $m$ and quartic dependence on $L$.

e. Charge mobility: According to Eq. (55) the charge mobility is independent of mass and can only be changed by varying the size of the box $L$. To model mobility in Fig. 6 , we evaluate $\mu_{e}$ using Eq. (40) for different values of $L, m$, and $\mathcal{E}$. To determine the average momentum, $\psi(x, t)$ was calculated using the variational approach. To ensure that we picked times of high momentum, we use $t$ from Eq. (54) with $k=1$, maximizing Eq. (53) where $n=2$. The resulting discrete values were plotted along with the solutions to Eq. (55), and their clear similarity shows that as long as $\mathcal{E}$ is sufficiently small, the mobility is quadrat- 


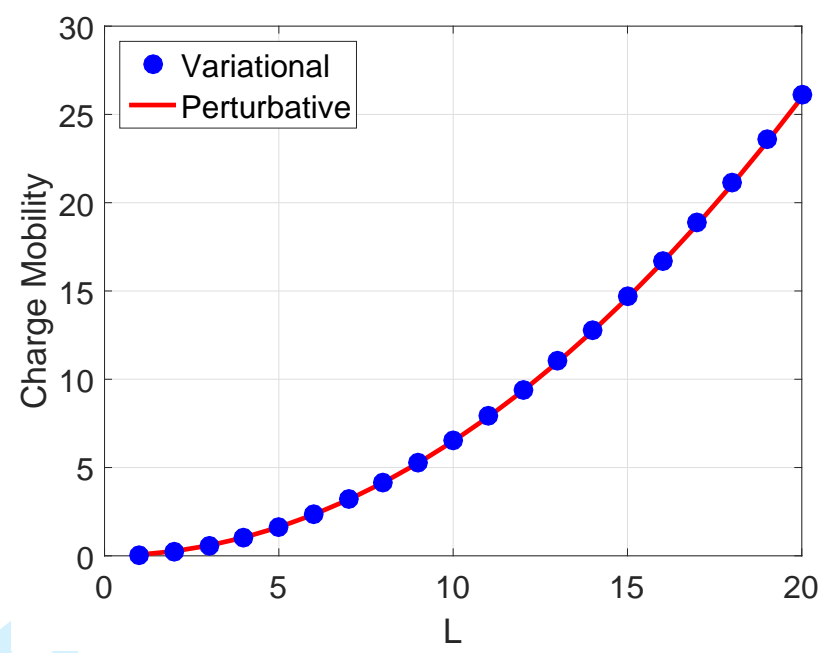

FIG. 6. Charge mobility dependence on the length of the box, for $\mathcal{E}=0.001$ and $m=0.1$. The variational results were obtained using the first 15 eigenfunctions.

ically dependent on $L$. In agreement with the perturbative prediction, variational calculations confirmed mobility independence from $m$ and $\mathcal{E}$, and thus were not included in this consideration.

\section{CONCLUDING REMARKS}

For a quantum particle to change its probability density in time, the particle needs to be in a superposition state. This is one of the most important concepts that underlies the phenomenon of conductivity. The particle-in-a-box problem with an inclined bottom can be used to illustrate this basic quantum mechanical principle behind electric conductivity. The key element in this phenomenon is that electrons in conductive materials are in the nonstationary state after the application of an electric field. The crucial system parameter for an efficient creation of this non-stationary (superposition) state is the gap between ground and excited electronic states. The larger the gap, the larger amount of energy required to create the superposition state necessary for the particle transport.

The particle-in-a-box model is the simplest model system where this dependence can be illustrated. This model is a useful example for lecture demonstrations using computer programs, for example MatLab.[9] It can also serve as a source of computer-assisted problems for computer-literate undergraduate students to obtain hands-on experience with the 
variational approach and perturbation theory.

\section{ACKNOWLEDGEMENTS}

A.F.I. is grateful to Al-Amin Dhirani, Dvira Segal, and Mark Ratner for stimulating discussions and acknowledges funding from the Natural Sciences and Engineering Research Council of Canada (NSERC) through the Discovery Grants Program and the Alfred P. Sloan Foundation.

[1] M. D. Fayer, in Absolutely Small: How Quantum Theory Explains Our Everyday World (Amacom, New York, 2010) p. 329.

[2] T. Engel, in Quantum Chemistry and Spectroscopy (Pearson, Boston, 2013) p. 73.

[3] J. M. Ziman, in Electrons and Phonons: The Theory of Transport Phenomena in Solids (Oxford at the Clarendon Press, Oxford, 1960) p. 92.

[4] N. W. Ashcroft and N. D. Mermin, in Solid State Physics (Saunders College, Orlando, Florida, 1976) p. 214.

[5] I. Wolfram Research, "Mathematica, version 10.4," Wolfram Research, Inc., Champaign, Illinois (2016).

[6] W. Kutzelnigg, Journal of Computational Chemistry 28, 25 (2006).

[7] D. J. Tannor, in Introduction to Quantum Mechanics: A Time-Dependent Perspective (University Science Books, Sausalito, California, 2007) p. 35.

[8] B. Fornberg, Mathematics of Computation 51, 699 (1988).

[9] "Matlab, version 8.0.0.783 (r2012b)," The MathWorks Inc., Natick, Massachusetts (2012). 